\title{
Occurrence of thymic pathology in two families. Is familial screening justified?
}

\author{
Sotirios D. Moraitis1, Apostolos C. Agrafiotis',2, Amalia Kapranou³, Marina Constantinou³, \\ Konstantinos Kanakakis1, Panagiotis Hountis1
}

\author{
1 Department of Thoracic Surgery, Athens Naval and Veterans Hospital, Athens, Greece \\ 2 Department of Thoracic Surgery, Tenon University Hospital, Paris, France \\ 3 Departement of Pathology, Athens Naval and Veterans Hospital, Athens, Greece
}

\begin{abstract}
The familial occurrence of thymic pathology, even though rare, is widely reported in the literature and mainly concerns cases of familial autoimmune myasthenia gravis. Other less frequent cases of familial occurrence of thymoma, thymic carcinoid and thymic hyperplasia have been described. It seems that the familial occurrence is poorly recorded and thus its prevalence is underestimated. We report two families whose members presented different forms of thymic pathology and discuss the necessity of screening programs in family members of patients presenting a thymic lesion.
\end{abstract}

\section{Introduction}

The familial occurrence of thymic pathology, even though rare, is widely reported in the literature and mainly concerns cases of familial autoimmune myasthenia gravis (MG) [1-9]. Other less frequent cases of familial occurrence of thymoma, thymic carcinoid and thymic hyperplasia have been described, as well [10-19]. It seems that the familial occurrence is poorly recorded and thus its prevalence is underestimated [1]. The genetic factors that may contribute to this familial clustering are not well known $[14,17]$. We report two families whose members presented different forms of thymic pathology. In the first case a

Corresponding author: Dr. Apostolos Agrafiotis, Department of Thoracic Surgery, Tenon University Hospital, 4 rue de la Chine, 75020 Paris, France. E-mail: apostolos.agrafiotis@aphp.fr

Key words: Familial; thymoma; thymic carcinoma; thymic hyperplasia.

Contributions: All the authors contributed equally to this work.

Conflict of interest: The authors declare no conflict of interest.

Received for publication: 3 February 2018

Accepted for publication: 1 August 2018

(C) Copyright S.D. Moraitis et al., 2018

Tipografia PI-ME Editrice, Italy

Monaldi Archives for Chest Disease 2018; 88:913

doi: $10.4081 /$ monaldi.2018.913

This article is distributed under the terms of the Creative Commons Attribution Noncommercial License (by-nc 4.0) which permits any noncommercial use, distribution, and reproduction in any medium, provided the original author(s) and source are credited. male patient developed a thymic carcinoma and his sister a thymoma (histological type A according to the WHO classification). In the second case, a female patient and her son presented anterior mediastinal lesions which were proved to be a thymic hyperplasia. In the case of the female patient, the thymic lesion was associated with limb muscle weakness, perhaps in the context of a seronegative myasthenia gravis.

\section{Case Reports}

\section{Family 1}

A 73-year-old male patient was diagnosed with an anterior mediastinal mass during a radiological workup of a lower respiratory system infection. The 18-Fluorodeoxyglycose Positron Emission Tomography (18FDG PET) scan showed a hypermetabolism of the lesion with a Standard Uptake Value $\left(\mathrm{SUV}_{\max }\right)$ of 4.6. The $\mathrm{SUV}_{\max }$ of the surrounding mediastinal structures was 1.6 (Figure 1a). There was no other abnormal intrathoracic or distant fixation. There were no clinical or biological evidence for myasthenia gravis or other paraneoplastic manifestation. The performed percutaneous transthoracic needle biopsy revealed a thymic carcinoma and the tumor board decision was in favor of a neoadjuvant chemotherapy and radiotherapy for initial downstaging. Presenting poor results in terms of mass reduction, the patient underwent a thymectomy via median sternotomy (Figure 1b). A tumor measuring $6 \times 5.5 \times 5 \mathrm{~cm}$ attached to two pieces of thymic fatty tissue of $7 \times$ $6 \times 1.5 \mathrm{~cm}$ and weight of $110.8 \mathrm{gr}$ was removed (Figure 1c). There was no invasion of the surrounding mediastinal structures or the lungs. The pathology examination revealed a complete (R0) resection of a thymic carcinoma with widespread degeneration and calcification (Figure 1d). Immunohistochemical examination was positive for Pankeratin (Figure 1e). The tumor board decided to further treat the patient with adjuvant chemo-radiotherapy.

A few months earlier, his 68-year old sister, who suffered Parkinson and Alzheimer diseases, was diagnosed with an anterior mediastinal mass, which was in fact a fortuitous discovery (Figure 2a). The 18FDG PET scan showed a hypermetabolism of the lesion with a $\mathrm{SUV}_{\max }$ of 3.6 , while the surrounding mediastinal structures presented a $\mathrm{SUV}_{\max }$ of 3.3. No other abnormal intrathoracic or distant fixation was revealed. The acetylcholine receptor (AchR) serum antibody test and Muscle-specific kinase receptor antibody (anti-MuSK) test were negative. A welldefined solid mass of $11.5 \times 10 \times 5.5 \mathrm{~cm}$, having a weight of $292 \mathrm{gr}$, attached to fatty tissue with dimensions of $8 \times 4 \times 2.5 \mathrm{~cm}$, was removed, through median sternotomy (Figure 2b). There was no invasion of the surrounding mediastinal structures or the lungs. The pathology examination showed a type A thymoma, according to the WHO classification; stage IIa, according to the Masaoka-Koga classification and pT2, according to TNM classification (Figure 2c). Further immunohistochemical investigation revealed neoplastic cells population being: $\mathrm{AE} \mathrm{1(+),}$ 
AE 3(+), Ker 20(-), Ker 7 (+), p63 (+), TTF1 (-), CD5(-) and Ki67 (+) at $10 \%$ of the nuclei of neoplastic cells (Figure $2 \mathrm{~d}$ ). A complete resection (R0) had been performed. The tumor board suggested continuing the treatment with radiotherapy.

\section{Family 2}

A 51-year-old female patient and her 27-year-old son (some months earlier) had the fortuitous discovery of anterior mediastinal lesions. The 27-year-old patient underwent a Computed Tomography (CT) scan during the investigation of a lower respiratory system infection which showed a lesion of the upper anterior mediastinum, at the level of the thymus. The ${ }^{18}$ FDG PET scan demonstrated a $\mathrm{SUV}_{\max }$ of 1.7, compared to the SUVmax of the mediastinal vessels that was 1.9. The patient became very concerned about the lesion and asked to undergo surgical removal, instead of biopsy or watchful attitude. He underwent a right thoracoscopic complete thymectomy and a hyperplastic thymus gland of $11.7 \times 9.1 \times 1.2 \mathrm{~cm}$ and $22.6 \mathrm{gr}$, was removed (Figure $3 \mathrm{a}$ ). The pathological examination confirmed thymic hyperplasia and did not reveal any neoplastic cells (Figure 3b). The 51-year-old female patient started complaining of physical tiredness and limb muscle weakness, after her son's operation. There was no biological evidence for myasthenia gravis. The acetylcholine receptor (AchR) serum antibody test and Muscle-specific kinase receptor antibody (anti-MuSK) test were negative. The performed CT and 18FDG PET scan showed the presence of a lesion at the upper anterior mediastinum, with a $\mathrm{SUV}_{\max }$ of 1.9 , compared to the $\mathrm{SUV}_{\max }$ of the surrounding mediastinal tissues that was 2.25. She underwent a right thoracoscopic complete thymectomy. A thymic remnant of $8.5 \times 4 \times 1.7 \mathrm{~cm}$ and $20 \mathrm{gr}$ was sent for pathology examination which revealed a thymic hyperplasia (Figure $3 \mathrm{c}, \mathrm{d}$ ). It is noteworthy that postoperatively her symptomatology disappeared.

\section{Discussion}

The majority of cases and case series of familial thymic disorders concern the autoimmune MG [1-9]. A Spanish study of familial autoimmune MG showed a prevalence of $3.46 \%$ [1]. The results of this cohort were compatible with the findings of other studies that concluded that familial autoimmune MG occurs in $1-5 \%$ of patients. However, it is advocated that its prevalence is higher, possibly due to an inadequate identification and recording of familial cases. The eventual genetic factors contributing to the familial form are not well known. The clinical manifestations, age of onset and status of serum antibodies may vary between family members [1,3]. Other autoimmune diseases may be present as well [1,7].

There are cases of familial autoimmune MG associated with a thymoma $[1,10]$. In the Spanish cohort there were two thymomas and five thymic hyperplasias among the myasthenic patients that underwent a thymectomy [1]. The first case of familial MG due to a thymoma in two
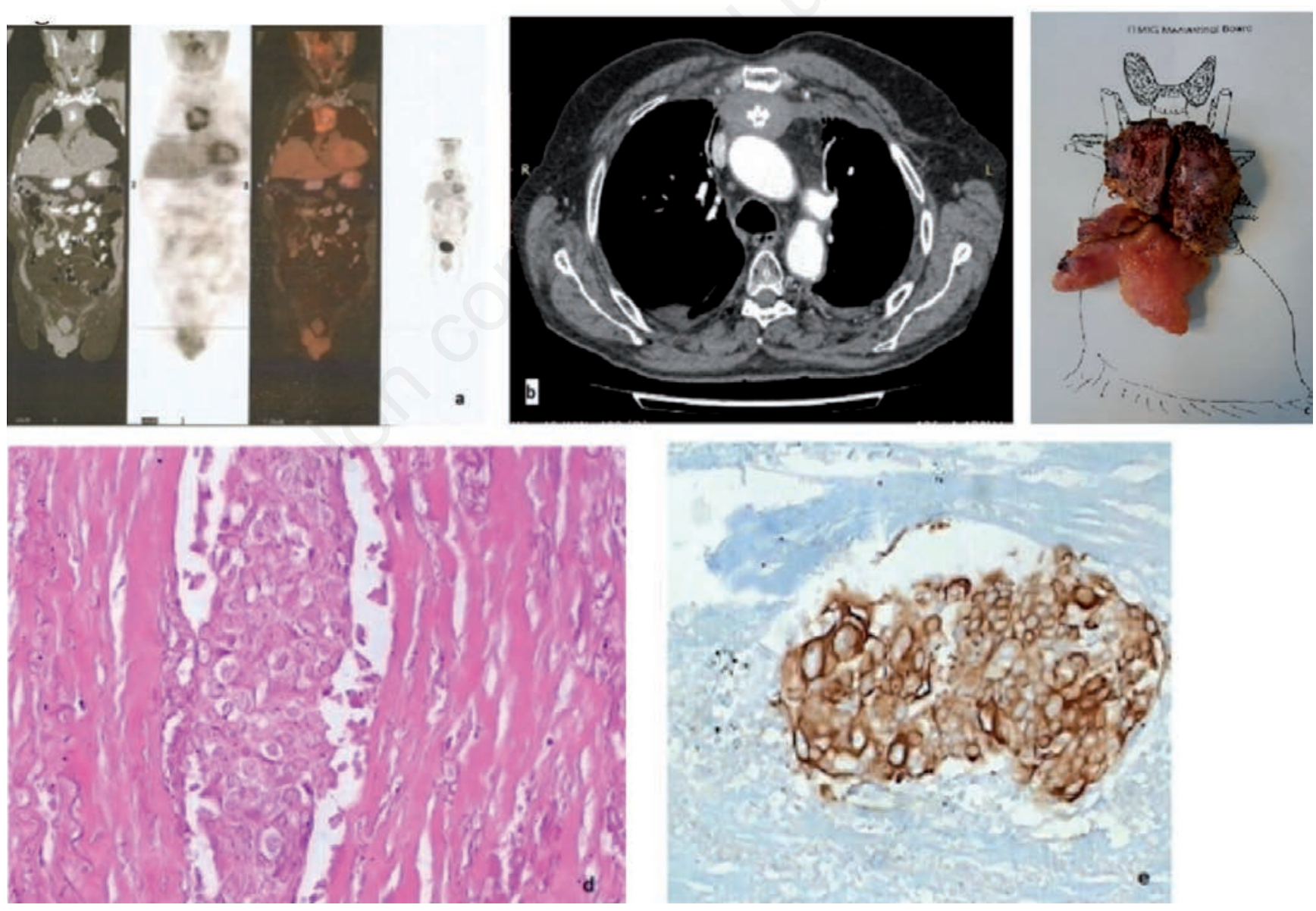

Figure 1. a) PET scan image showing the hypermetabolism of the anterior mediastinal mass; b) CT scan image showing the anterior mediastinal mass after neoadjuvant chemoradiotherapy; c) Pathologic specimen after surgical resection; d) H/E x400 image of a thymic carcinoma; e) Pankeratin (+) thymic carcinoma. 
brothers was reported in 1986 [18]. On the other hand, the familial occurrence of thymoma without MG was first reported in 1968 [11]. Matani and Dritsas in 1973 described a thymoma that was the cause of death of a 27-month-old girl [12]. Two years earlier her older brother died at the age of 9 months due to a respiratory failure. The post mortem examination revealed a thymoma. The prevalence of familial thymoma is estimated between 1 and 4 per million and its cause is also unknown [13-17]. Familial thymomas can be associated with other malignancies and autoimmune diseases [14,17]. Zhang et al. suggest that the association of familial breast cancer and thymoma could constitute a cancer syndrome [17].

Lim et al. reported a MEN1 family in which two members developed malignant thymic carcinoids [19]. The authors discussed the necessity of prophylactic thymectomy in patients presenting this syndrome, taking into consideration the aggressiveness of thymic carcinoids. However, the genetic basis of familial thymic carcinoid is not yet established.

The presence of human polyomavirus 7 (HPyV7) in adult thymic epithelial tumors could possibly link this virus to the pathogenesis of human thymomas, however its exact role has to be established [20, 21]. In our institution, the pathology department is not supplied with DNA Fluorescence in situ hybridization (FISH) and DNA-polymerase chain reaction (PCR) as diagnostic methods. Moreover, we cannot detect human polyomavirus 7 by immunohistochemistry, as we don't have the special monoclonal antibody 2T10 against LTAg of HPyV7.

It has to be highlighted that neither of the families presented herein had a history of MG. The female patient on the second family, presented limb muscle weakness that disappeared after surgery, but no other preoperative tests were performed in order to formally call it seronegative MG. Certainly, the coexistence of thymic pathology in these two families could be a coincidence and not genetically related. We must take into consideration the fact that genetic factors are difficult to investigate in order to prove this relationship. On the other hand we must admit that the frequency of this kind of pathology among family members is poorly identified and recorded, especially in asymptomatic subjects compared to myasthenic ones. Even though thymic pathology is rare, in our opinion screening should be encouraged in family members of these patients. Despite the lack of efficacy and cost-effectiveness data, we could exhort all adult family members to undergo an acetylcholine receptor (AchR) serum antibody test, a Muscle-specific kinase receptor antibody (anti-MuSK) test and a contrast-enhanced chest CT scan, as baseline examinations. In case of negative CT scan, this could be repeated after 5 years, as sometimes the growth of thymic lesions can be slow [22]. Specific registries for
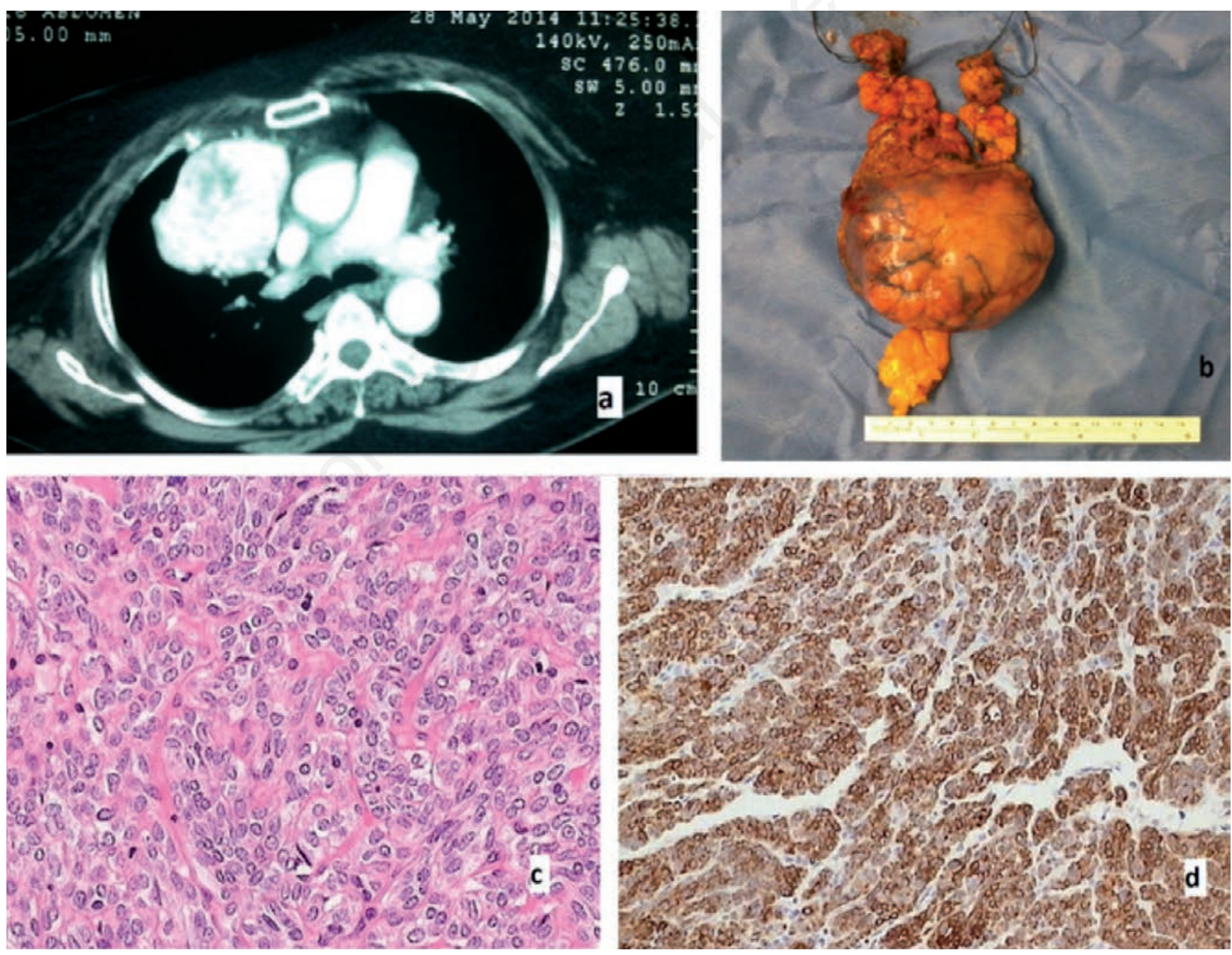

Figure 2. a) CT scan image showing the anterior mediastinal mass; b) surgical specimen; c) H/E x400 image of a type A thymoma; d) pankeratin (+) type A thymoma. 

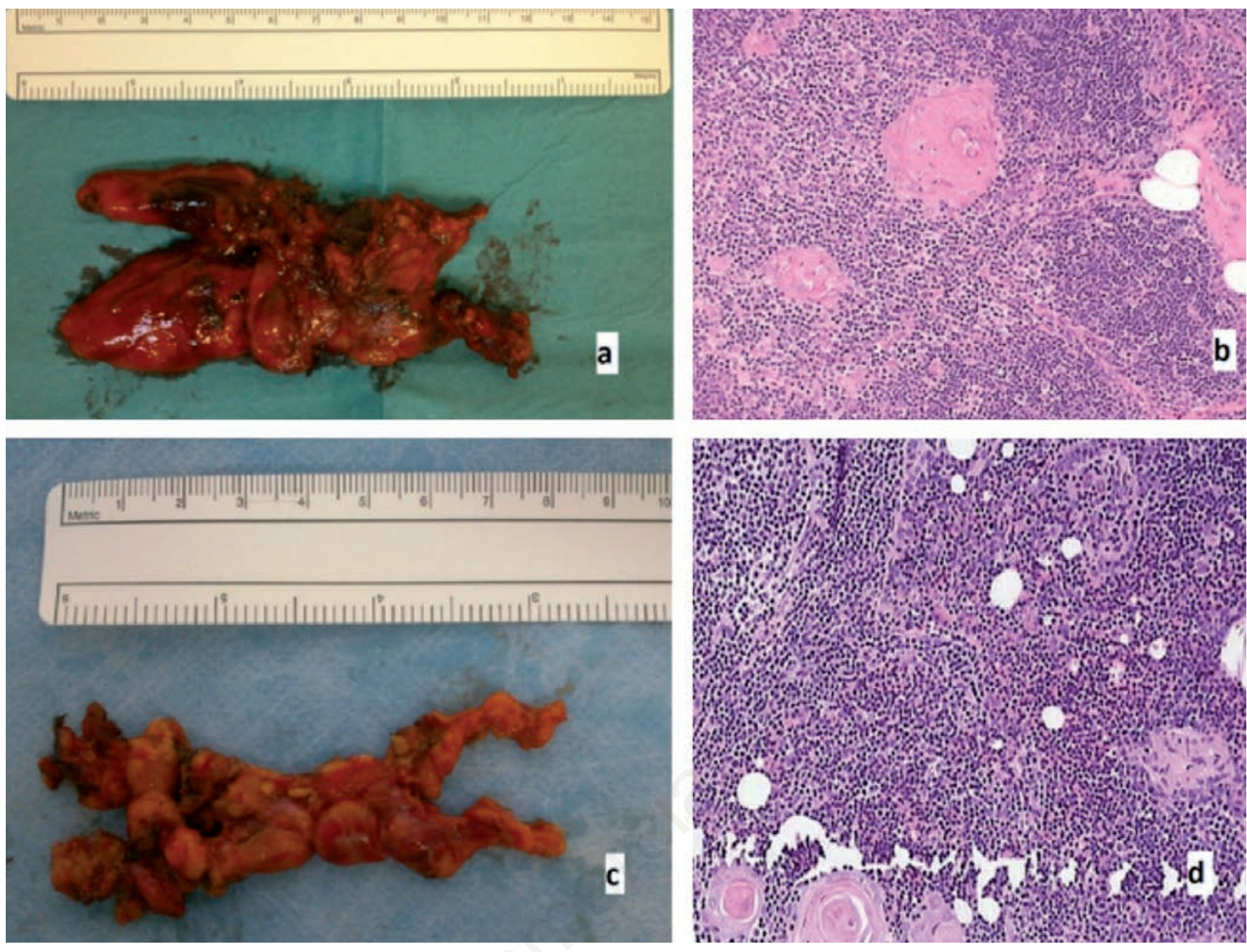

Figure 3. a) Surgical specimen of a thymic hyperplasia; b) H/E x200 image of a thymic hyperplasia; c) surgical specimen of a thymic remnant in the mother of the previous patient; d) H/E x200 image of a thymic hyperplasia.

these rare pathologies could help better record their incidence and establish the role of such a screening protocol, while defining the appropriate frequency of follow up.

\section{Conclusions}

Familial occurrence of thymic pathology is a condition that is difficult to identify especially in asymptomatic patients. For that reason its prevalence is probably underestimated. Further studies should be conducted in order to identify possible genetic factors and investigate the necessity of screening programs in family members of patients presenting a thymic lesion.

\section{References}

1. Salvado M, Canela M, Ponseti JM, et al. Study of the prevalence of familial autoimmune myasthenia gravis in a Spanish cohort. J Neurol Sci 2016;360:110-4.

2. Marrie RA, Sahlas DJ, Bray JM. Familial autoimmune myasthenia gravis: four patients involving three generations. Can J Neurol Sci 2000;27:307-10.
3. Chen Y, Wang W, Wei D, Yang L. Three cases of myasthenia gravis from one family with variations in clinical features and serum antibodies. Neuromusc Disord 2012;22:286-8.

4. Evoli A, Batocchi AP, Zelano G, et al. Familial autoimmune myasthenia gravis: report of four families. J Neurol Neurosurg Psychiatry 1995;58:729-31.

5. Honeybourne D, Dyer PA, Mohr PD. Familial myasthenia gravis. J Neurol Neurosurg Psychiatry1982;45:854-6.

6. Szobor A. Familial myasthenia gravis:nine patients in two generations. Acta Med Hung 1991;48:145-9.

7. Wright R, Kerr JH. Autoimmunity in myasthenia gravis:a family study. Clin Exp Immunol 1967;2:93-101.

8. Khadilkar SV, Shah SV. Autoimmune myasthenia gravis in two brothers. J Assoc Physicians India 2004;52:253-4.

9. Namba T, Brunner NG, Brown SB, Muguruma M, et al. Familial myasthenia gravis. Report of 27 patients in 12 families and review of 164 patients in 73 families. Arch Neurol 1971;25:49-60.

10. Rotstein DL, Bril V. A family with myasthenia gravis with and without thymoma. Can J Neurol Sci 2012;39:539-40.

11. Slowikowski J, Kubrakiewicz Z, Zielinski S. Giant hypertrophy of the thymus in three siblings. Pol Med J 1968;634-7.

12. Matani A, Dritsas C. Familial occurrence of thymoma. Arch Pathol 1973;95:90-1. 
13. Deminatti MM, Ribet M, Gosselin B, et al. Familial thymoma and translocation t (14;20) (q24;p13). Ann Genet 1994;37:72-4.

14. Nicodeme F, Geffroy S, Conti M, et al. Familial occurrence of thymoma and autoimmune diseases with the constitutional translocation t (14;20) (q24.1;p12.3). Genes Chromosomes Cancer 2005;44:154-60.

15. Wick MR, Scheithauer BW, Dines DE. Thymic neoplasia in two male siblings. Mayo Clin Proc 1982;57:653-6.

16. Lam WW, Chan FL, Lau YL, et al. Paediatric thymoma: unusual occurrence in two siblings. Pediatr Radiol 1993;23:124-6.

17. Zhang X, Wang T, Wang W, et al. Does familial breast cancer and thymoma suggest a cancer syndrome? A family perspective. Gene 2015;573:333-7.

18. Pascuzzi RM, Sermas A, Phillips LH 2nd, Johns TR. Familial autoimmune myasthenia gravis and thymoma: occurrence in two brothers. Neurology 1986;36:423-7.

19. Lim LC, Tan MH, Eng C, et al. Thymic carcinoid in multiple endocrine neoplasia 1: genotype-phenotype correlation and prevention. J Intern Med 2006;259:428-32.

20. Rennspiess D, Pujari S, Keijzers M, et al. Detection of human polyomavirus 7 in human thymic epithelial tumors. J Thorac Oncol 2015;10:360-6.

21. Keijzers M, Rensspiess D, Pujari S, et al. Expression of $\mathrm{pRb}$ and p16INK4 in human thymic epithelial tumors in relation to the presence of human polyomavirus 7. Diagn Pathol 2015;10:201.

22. Rashid OM, Cassano AD, Takabe K. Thymic neoplasm: a rare disease with a complex clinical presentation. J Thorac Dis 2013;5:173-83. 